\title{
RELATIONSHIPS BETWEEN REDUCIBILITIES $\left({ }^{1}\right)$
}

\author{
CARL G. JOCKUSCH, JR.
}

1. Introduction. We shall consider the following recursion theoretic reducibilities: one-one (1), many-one $(m)$, truth-table $(t t)$, and Turing $(T)$. These were introduced by Post [13] and are treated also by Rogers in [15]. If $R$ is one of these reducibilities, we write $A \leqq_{R} B$ if $A$ is $R$-reducible to $B$ and $A \equiv_{R} B$ if $A \leqq_{R} B$ and $B \leqq_{R} A$. Also we write $B \varliminf_{R} A$ if $B \leqq_{R} A$ is false, and $A<_{R} B$ if $A \leqq_{R} B$ and $B \varliminf_{R} A$. For any set $B$, the collection of all $A$ such that $A \equiv_{R} B$ is an $R$-degree. An $R$-degree is r.e. (recursive) if it contains at least one r.e. (recursive) set.

Myhill [11, Theorems 5 and 16] proved that the $m$-degree of the creative sets consists of a single 1-degree. Young [18, Corollary 1.5] showed that every nonrecursive $m$-degree either consists of a single 1-degree or contains a collection of 1-degrees having the order type of the rationals. He asked whether there exist r.e. nonrecursive $m$-degrees consisting of a single 1-degree other than the $m$-degree of the creative sets. We show here that the answer is affirmative and indeed that each r.e. $T$-degree contains an r.e. $m$-degree consisting of a single 1-degree. (This has also been proved by Soare [16, Corollary 4.6].) We also show that each r.e. nonrecursive $T$-degree contains infinitely many r.e. $m$-degrees and each nonrecursive $t t$-degree contains infinitely many $m$-degrees. Finally we show that each $T$-degree either consists of a single $t t$-degree or contains infinitely many $t t$-degrees.

We assume that the collection of all finite sets has been effectively coded onto the set of natural numbers, and we write $D_{x}$ for the finite set with index $x$. We freely identify $x$ and $D_{x}$, and if $\mathscr{A}$ is a collection of finite sets, we often view $\mathscr{A}$ as $\left\{x: D_{x} \in \mathscr{A}\right\}$. If $A$ is a set, we write $\mathscr{A}^{\omega}$ for $\left\{D_{x}: D_{x} \subset A\right\}$.

If $g$ is a recursive function such that for all $x, x \in C \Leftrightarrow g(x) \in B$, we say $C \leqq_{m} B$ via $g$. We say that the array $D_{f(x)}$ witnesses that $B$ is not hyperimmune if $f$ is a recursive function, $D_{f(x)} \cap D_{f(u)}=\varnothing$ for $x \neq u$, and $D_{f(x)} \cap B \neq \varnothing$ for all $x$. We write $\bar{A}$ for the complement of the set $A$ and $\left|D_{x}\right|$ for the cardinality of the finite set $D_{x}$.

2. R.e. $m$-degrees and 1-degrees within r.e. $T$-degrees. The following technical lemma will be useful in the proof of both theorems of this section.

Lemma 1. Suppose $A$ is simple but $D_{f(x)}$ witnesses that $\bar{A}$ is not hyperimmune.

Received by the editors August 26, 1968.

(1) The first three sections of this paper are adapted from the author's doctoral thesis [5] written under the direction of Professor H. Rogers, Jr. Preparation of this paper was supported by NSF Grant GP 7421 at the University of Illinois. 
Then there is no recursive class $\mathscr{C}$ of nonempty finite sets such that each $D_{f(x)}$ belongs to $\mathscr{C}$ and for all $x, y$ :

(i) $D_{x} \in \mathscr{C}, y \in A \Rightarrow\left(D_{x}-\{y\}\right) \in \mathscr{C}$.

Proof. Assume there were such a class $\mathscr{C}$. Let $\mathscr{M}$ be the collection of all finite sets which belong to $\mathscr{C}$ but have no proper subset which belongs to $\mathscr{C}$. Let $G=\bigcup \mathscr{M}$, the union of all the sets in $\mathscr{M}$. It is claimed that $G$ is an infinite r.e. subset of $\bar{A}$. If this claim is proved, the simplicity of $A$ will be contradicted.

Since $\mathscr{C}$ is recursive, $\mathscr{M}$ is recursive, and thus $G$ is r.e. Now any member of $\mathscr{C}$ which intersects $A$ has a proper subset in $\mathscr{C}$ by condition (i). Hence every set in $\mathscr{M}$ is contained in $\bar{A}$, so $G \subset \bar{A}$. Finally note that each set $D_{x} \in \mathscr{C}$ has a subset $D_{z} \in \mathscr{M}: D_{z}$ is any subset of $D_{x}$ of minimal cardinality among the subsets of $D_{x}$ which belong to $\mathscr{C}$. Thus each $D_{f(x)}$ has a subset in $\mathscr{M}$ and therefore $G$ intersects each $D_{f(x)}$, since all members of $\mathscr{C}$ are nonempty. Hence $G$ is infinite.

THEOREM 2. If $A$ is simple but not hypersimple and $\mathscr{A}^{\omega} \leqq_{m} B$, then $\mathscr{A}^{\omega} \leqq_{1} B$.

Proof. Assume $\mathscr{A}^{\omega} \leqq_{m} B$ via the recursive function $g$, i.e. for all $x, D_{x} \subset A$ $\Leftrightarrow g(x) \in B$. Consider a propositional language $\mathscr{L}$ with all the standard connectives in which the statement letters are " $y \in A$ " and " $y \in B$ " for each number $y$. The assumptions that have been made can be represented in $\mathscr{L}$ by the following infinite but r.e. set of axioms $T$ :

$$
\begin{array}{ll}
D_{x} \cap \bar{A} \neq \varnothing \leftrightarrow g(x) \notin B & \text { all } x . \\
y \in A & \text { all } y \in A . \\
D_{f(x)} \cap \bar{A} \neq \varnothing & \text { all } x .
\end{array}
$$

(Any statement above which is not a formula of $\mathscr{L}$ should be interpreted in the obvious way. For instance, if $D_{f(0)}=\{2,5\}$, then " $D_{f(0)} \cap \bar{A} \neq \varnothing$ " is rendered in $\mathscr{L}$ by " $\neg 2 \in A \vee \neg 5 \in A$ ". Note also that we use $\leftrightarrow$ for equivalence in $\mathscr{L}$ while $\Leftrightarrow$ is used metalinguistically.)

If $\phi$ is (an abbreviation for) a formula of $\mathscr{L}, \vdash_{T} \phi$ means that $\phi$ can be proved from the hypotheses $T$ within the propositional calculus.

For each number $k$, let

$$
E_{k}=\left\{y: \vdash_{T} D_{k} \subset A \leftrightarrow y \in B\right\} .
$$

We assume temporarily that $E_{k}$ is infinite for every $k$ and show how the theorem then follows. Notice that the sets $E_{k}$ are r.e. uniformly in $k$. To define the recursive function $h$ by induction, let $h(k)$ be the first number $y$ which occurs in a uniform enumeration of $E_{k}$ such that $y \neq h\left(k^{\prime}\right)$ for all $k^{\prime}<k$. $h$ is total because $E_{k}$ is always infinite, and so $\mathscr{A}^{\omega} \leqq_{1} B$ via $h$ by the soundness of propositional logic.

It remains to show that each $E_{k}$ is infinite. We fix $k$ and assume that $E_{k}$ is finite.

Consider first the case where $\vdash_{T} D_{k} \cap \bar{A} \neq \varnothing$. Then $E_{k}=\left\{y: \vdash_{T} y \notin B\right\}$. Thus. by axioms $\left(1_{x}\right), \vdash_{T} D_{x} \cap \bar{A} \neq \varnothing$ iff $g(x) \in E_{k}$, for any $x$. Hence if $\mathscr{C}=\left\{D_{x}: \vdash_{T} D_{x}\right.$ $\cap \bar{A} \neq \varnothing\}, \mathscr{C}$ is recursive. Also $\mathscr{C}$ contains only nonempty sets by the soundness of propositional logic, and all the sets $D_{f(x)}$ belong to $\mathscr{C}$ by axioms $\left(3_{x}\right)$. Finally, 
condition (i) of Lemma 1 is satisfied for $\mathscr{C}$ because of axioms $\left(2_{y}\right)$. Hence the assumption that $E_{k}$ was finite has led to a contradiction in this case.

Now assume that $\nvdash_{T} D_{k} \cap \bar{A} \neq \varnothing$. (We write " $\nvdash_{T} \phi$ " for "not $\vdash_{T} \phi$.") Let

$$
\mathscr{C}_{k}=\left\{D_{x}: \nvdash_{T} D_{x} \cup D_{k} \subset A \leftrightarrow D_{k} \subset A\right\} .
$$

Clearly $\mathscr{C}_{k}$ consists only of nonempty sets. Also $\mathscr{C}_{k}$ contains all the sets $D_{f(x)}$ because if $D_{f(x)} \notin \mathscr{C}_{k}$ for some particular $x$, then $\vdash_{T} D_{f(x)} \cup D_{k} \subset A \leftrightarrow D_{k} \subset A$ and so $\vdash_{T} D_{k} \cap \bar{A} \neq \varnothing$ by axiom $\left(3_{x}\right)$. To see that $\mathscr{C}_{k}$ satisfies condition (i) of Lemma 1 we assume that $y \in A$ and $\left(D_{x}-\{y\}\right) \notin \mathscr{C}_{k}$ and prove that $D_{x} \notin \mathscr{C}_{k}$. Since $D_{x}-\{y\}$ $\notin \mathscr{C}_{k}$,

$$
\vdash_{T}\left(D_{x}-\{y\}\right) \cup D_{k} \subset A \leftrightarrow D_{k} \subset A .
$$

Since $y \in A$ we see from axiom $(2 y)$ that

$$
\vdash_{T} D_{x} \cup D_{k} \subset A \leftrightarrow D_{k} \subset A,
$$

i.e. $D_{x} \notin \mathscr{C}_{k}$.

To verify that $\mathscr{C}_{k}$ is recursive we apply axioms $\left(2_{x}\right)$ to the definition of $\mathscr{C}_{k}$ and deduce that for all $x$,

$$
D_{x} \in \mathscr{C}_{k} \Leftrightarrow \nvdash_{T} g\left(D_{x} \cup D_{k}\right) \in B \leftrightarrow D_{k} \subset A .
$$

Thus $D_{x} \in \mathscr{C}_{k}$ iff $g\left(D_{x} \cup D_{k}\right) \notin E_{k}$. Since $E_{k}$ is finite, it follows that $\mathscr{C}_{k}$ is recursive and we have a contradiction to Lemma 1 .

The following corollary has been proved independently by Soare [16, Corollary 4.6].

COROllaRY 3. Each r.e. T-degree contains an r.e. m-degree which consists of a single 1-degree.

Proof. In the recursive $T$-degree, the $m$-degree of $\varnothing$ consists of a single 1-degree. By a theorem of Yates, [17, Theorem 2], each nonrecursive r.e. $T$-degree $a$ contains a simple nonhypersimple set $A$. Then $\mathscr{A}^{\omega}$ is also an r.e. set of degree $a$, and we claim that the $m$-degree of $\mathscr{A}^{\omega}$ consists of a single 1-degree. Assume $\mathscr{A}^{\omega} \equiv_{m} B$. By Theorem $2, \mathscr{A}^{\omega} \leqq_{1} B$. Because $A$ is an infinite r.e. set it is easy to see from Lemma 0.5 of [18] that $\mathscr{A}^{\omega}$ is a cylinder. It then follows from [15, Theorem VIII(c)] that $B \leqq_{1} \mathscr{A}^{\omega}$. Thus $B \equiv_{1} \mathscr{A}^{\omega}$ and we have the desired conclusion.

We remark that in Theorem $2, \mathscr{A}^{\omega}$ may be replaced by $\mathscr{A}^{\omega} \times B$, where $B$ is any nonempty set. $(C \times B$, for sets $C, B$, is the image of the cartesian product of $C$ and $B$ under a recursive pairing function.) The proof of Theorem 2 remains essentially the same. The reasoning of the proof of Corollary 3 then shows that each $T$-degree which has a nonrecursive r.e. predecessor has an $m$-degree consisting of a single 1-degree. We do not know whether all $T$-degrees have an $m$-degree consisting of a single 1-degree.

If $A$ is a set, we let $\mathscr{A}^{n}=\left\{D_{x}: D_{x} \subset A \&\left|D_{x}\right| \leqq n\right\}$. Note that $\mathscr{A}^{n} \leqq_{m} \mathscr{A}^{n+1}$ for all $n$. The theorems we shall state for $\mathscr{A}^{n}$ we could equally well state for $A^{n}$ 


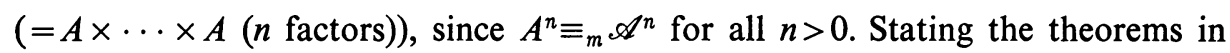
terms of $A^{n}$ is more conventional, but $\mathscr{A}^{n}$ is more convenient technically in the present context.

THEOREM 4. If $A$ is simple but not hypersimple, then for all $n, \mathscr{A}^{n}<_{m} \mathscr{A}^{n+1}$.

Proof. Assume, for reductio ad absurdum, that $\mathscr{A}^{n+1} \leqq_{m} \mathscr{A}^{n}$. Call two esets $E$, $E^{\prime} A$-equivalent if $E \subset A \Leftrightarrow E^{\prime} \subset A$. The assumption that $\mathscr{A}^{n+1} \leqq_{m} \mathscr{A}^{n}$ implies that given any set of cardinality $n+1$ one can effectively find an $A$-equivalent set of cardinality at most $n$. Thus given any finite set $E$ of cardinality $k>n$, one may effectively find an $A$-equivalent set $F$ of cardinality at most $k-1$ by replacing some $n+1$ element subset of $E$ by an $A$-equivalent set of at most $n$ elements. If this procedure is iterated $k-n$ times, one finds effectively a set of cardinality at most $n$ which is $A$-equivalent to $E$. Hence $\mathscr{A}^{\omega} \leqq_{m} \mathscr{A}^{n}$.

Assume $\mathscr{A}^{\omega} \leqq_{m} \mathscr{A}^{n}$ via $g$, where $\left|D_{g(x)}\right| \leqq n$ for all $x$. Assume that $D_{f(x)}$ witnesses that $\bar{A}$ is not hyperimmune. Let $\mathscr{L}$ be the propositional language which has statement letters " $y \in A$ " for each number $y$. Let $T$ be the following r.e. set of axioms in $\mathscr{L}$ :

$$
\begin{array}{ll}
D_{x} \cap \bar{A} \neq \varnothing \leftrightarrow D_{g(x)} \cap \bar{A} \neq \varnothing & \text { all } x . \\
y \in A & \text { all } y \in A . \\
D_{f(x)} \cap \bar{A} \neq \varnothing & \text { all } x .
\end{array}
$$

We use " $\vdash_{T} \phi$ " as in Theorem 2. For each $k$, let $\mathscr{A}_{k}=\left\{D_{x}:\left|D_{x}\right| \leqq k \& \vdash_{T} D_{x} \cap \bar{A}\right.$ $\neq \varnothing\}$. We prove that $\mathscr{A}_{k}$ is recursive by induction on $k . \mathscr{A}_{0}$ is the empty class. Now assume that $\mathscr{A}_{k}$ is recursive and consider $\mathscr{A}_{k+1}$. We claim that $\mathscr{A}_{k+1}$ contains only finitely many sets which have no subset in $\mathscr{A}_{k}$. The proof of this is similar to the proof of Lemma 1 . Let $\mathscr{M}$ be the collection of all sets in $\mathscr{A}_{k+1}$ which have no subset in $\mathscr{A}_{k}$ and let $G=\cup \mathscr{M} . \mathscr{M}$ is r.e. because $\mathscr{A}_{k+1}$ is r.e. and $\mathscr{A}_{k}$ is recursive. Hence $G$ is r.e. It is easy to show from axioms $2_{x}$ that $G \subset \bar{A}$. Hence $G$ is finite, so $\mathscr{M}$ is finite. Thus $\mathscr{A}_{k+1}$, with finitely many exceptions, consists just of those sets which have at most $k+1$ elements and contain some set in $\mathscr{A}_{k}$. Hence, by another application of the induction assumption, $\mathscr{A}_{k+1}$ is recursive.

Let $\mathscr{C}=\left\{D_{x}: \vdash_{T} D_{x} \cap \bar{A} \neq \varnothing\right\}$. By axiom $1_{x}, D_{x} \in \mathscr{C} \Leftrightarrow D_{g(x)} \in \mathscr{A}_{n}$. Since $\mathscr{A}_{n}$ is recursive, it follows that $\mathscr{C}$ is recursive. One many verify exactly as for $\mathscr{C}$ in Theorem 2 that $\mathscr{C}$ satisfies the other hypotheses of Lemma 1 . But Lemma 1 states that no such $\mathscr{C}$ exists, and so we have a contradiction.

Fischer [4, Theorem 2] first proved Theorem 4 (for $A^{n}$ ) in the special case where $A$ was Post's $t t$-complete simple set $[13$, p. 304]. However, our proof is entirely different from his.

COROLlaRY 5. Each nonrecursive r.e. T-degree contains an infinite chain of r.e. m-degrees. 
Proof. Let $\boldsymbol{a}$ be a given nonrecursive r.e. $T$-degree, and let $A$ be a simple nonhypersimple set of $T$-degree $a\left[17\right.$, Theorem 2]. Then the r.e. sets $\mathscr{A}^{1}, \mathscr{A}^{2}, \ldots$ also have $T$-degree $\boldsymbol{a}$ and $\mathscr{A}^{1}<_{m} \mathscr{A}^{2}<_{m} \ldots$ by the theorem.

We remark that the assumption of nonhypersimplicity cannot be dropped from either theorem of this section. In particular, it is easy to see from [6, Theorems 3.2 and 4.2 (i)] and well-known results that the conclusions of these theorems are false for any set $A$ obtained by Dekker's hypersimple set construction [2, Theorem $1]$.

It is natural to enquire whether an analogue of Corollary 5 holds for antichains, i.e. collections of mutually incomparable $m$-degrees. Along these lines, Lerman [8, Theorem 5.1] proved that each r.e. $T$-degree $\boldsymbol{a}$ with $\boldsymbol{a}^{\prime}=\mathbf{0}^{\prime \prime}$ has an infinite antichain of $m$-degrees of maximal sets. The $m$-degrees of maximal sets have the additional interesting property of being minimal among the nonrecursive $m$ degrees [7, p. 4] and [15, p. 239]. Lachlan [7, Theorem 4] has shown the existence of a minimal r.e. $m$-degree in each nonzero r.e. $T$-degree, but the existence of an infinite antichain of r.e. $m$-degrees in each such $T$-degree has not yet been established.

3. $m$-degrees within $t t$-degrees.

THEOREM 6. If $B$ is immune but not hyperimmune and retraced by a general recursive function and $A=\bar{B}$, then for all $n, \mathscr{A}^{n}<_{m} \mathscr{A}^{n+1}$.

Proof. Assume that $B$ is retraced by the general recursive function $h$. This means [3, p. 358] that if $b_{k}$ is the $k$ th smallest member of $B$, then $h\left(b_{0}\right)=b_{0}$ and $h\left(b_{k+1}\right)=b_{k}$ for all $k \geqq 0$. We may assume that $h(x) \leqq x$ for all $x$, i.e that $h$ is downward. The proof to follow may best be visualized in terms of the "retracing tree" of $h$. (See [3, p. 359].) Following Appel and McLaughlin [1, p. 83] we set $\hat{h}(y)=$ $\left\{h^{k}(y): k \geqq 0\right\}$. (Here $h^{0}(y)=y$ and $h^{k+1}(y)=h\left(h^{k}(y)\right.$.) Because $h$ is general recursive and downward, the predicate $z \in \hat{h}(y)$ is recursive.

We say that $y$ and $z$ are $h$-comparable if $y \in \hat{h}(z)$ or $z \in \hat{h}(y)$. If $y$ and $z$ both belong to $B, y$ and $z$ are $h$-comparable. We write $y \mid D_{x}$ if $y$ is not $h$-comparable with any member of $D_{x}$.

Let $A=\bar{B}$ and assume that $\mathscr{A}^{n+1} \leqq_{m} \mathscr{A}^{n}$ via $g$. We may assume that $\left|D_{g(x)}\right| \leqq n$ whenever $\left|D_{x}\right| \leqq n+1$, so we have for such $x$,

$$
D_{x} \subset \bar{B} \Leftrightarrow D_{g(x)} \subset \bar{B} .
$$

We now wish to define a certain r.e. set $C \subset \bar{B}$. Let $C=C_{1} \cup C_{2}$, where

$$
\begin{aligned}
& C_{1}=\left\{y:(\exists x)\left[\left|D_{x}\right| \leqq n+1 \& y \in D_{x} \& y \mid D_{g(x)}\right]\right\}, \\
& C_{2}=\left\{y:(\exists x)\left[\left|D_{x}\right| \leqq n+1 \& y \mid D_{x} \& \hat{h}(y) \cap D_{g(x)} \neq \varnothing\right]\right\} .
\end{aligned}
$$

$C_{1}$ and $C_{2}$ are r.e. so $C$ is r.e. To see that $C_{1} \subset \bar{B}$, suppose that some number $y$ were in $C_{1} \cap B$. Let $x$ be such that $\left|D_{x}\right| \leqq n+1$ and $y \in D_{x}$ and $y \mid D_{g(x)}$. Since $y \in B$ and $y \mid D_{g(x)}, D_{g(x)} \subset \bar{B}$. Thus also $D_{x} \subset \bar{B}$, but this is impossible since $y \in D_{x} \cap B$. 
To see that $C_{2} \subset \bar{B}$, suppose that some number $y$ were in $C_{2} \cap B$. Let $x$ be such that $\left|D_{x}\right| \leqq n+1$ and $y \mid D_{x}$ and $\hat{h}(y) \cap D_{g(x)} \neq \varnothing$. Since $y \in B$ and $y \mid D_{x}, D_{x} \subset \bar{B}$. Hence $D_{g(x)} \subset \bar{B}$. Since $y \in B, \hat{h}(y) \subset B$, so $\hat{h}(y) \cap D_{g(x)}=\varnothing$. This is a contradiction. Thus $C_{2} \subset \bar{B}$, so $C \subset \bar{B}$.

For any $y$, let the level of $y$ be the least number $k$ such that $h^{k}(y)=h^{k+1}(y)$. ( $k$ exists because $h$ is downward.) A set $D$ is level if all members of $D$ have the same level. We claim that $C$ is so large that $\bar{C}$ has no level subset of cardinality exceeding $2 n$. (Recall that $n$ was chosen so that $\mathscr{A}^{n+1} \leqq_{m} \mathscr{A}^{n}$.) To facilitate the proof of this claim, a partial ordering $<_{l}$ of the natural numbers will be defined such that any two numbers $y, z$ are $<_{l}$-comparable iff $y$ and $z$ have the same level. If $y$ and $z$ have level 0 , we define $y<{ }_{l} z$ iff $y<z$. If $y$ and $z$ have level $k+1$, then $h(y)$ and $h(z)$ have level $k$, and we assume inductively that we have defined $<_{l}$ for $h(y)$ and $h(z)$. We now define

$$
y<{ }_{l} z \Leftrightarrow h(y)<{ }_{l} h(z) \text { or }(h(y)=h(z) \& y<z) .
$$

(Thus if the retracing tree of $h$ is drawn appropriately, $y<_{l} z$ means that $y$ and $z$ have the same level and $y$ lies to the left of $z$.)

Clearly $<_{l}$ is a partial ordering. We write $y \leqq_{l} z$ if $y<_{l} z$ or $y=z$. If $y<_{l} z$, then for any $k, h^{k}(y) \leqq_{l} h^{k}(z)$.

Now suppose, contrary to our claim, that $\bar{C}$ has a level subset $\left\{y_{1}, y_{2}, \ldots, y_{2 n+1}\right\}$ of cardinality $2 n+1$. Assume $y_{1}<_{l} y_{2}<_{l} \cdots<_{l} y_{2 n+1}$, and let

$$
D_{x}=\left\{y_{1}, y_{3}, y_{5}, \ldots, y_{2 n+1}\right\} \text {. }
$$

Since no member of $D_{x}$ is in $C_{1}$, every member of $D_{x}$ is $h$-comparable with some member of $D_{g(x)}$. But since $\left|D_{x}\right| \leqq n+1,\left|D_{g(x)}\right| \leqq n$. Thus some member $z$ of $D_{g(x)}$ is $h$-comparable with two distinct members, say $y_{2 i+1}$ and $y_{2 j+1}$, of $D_{x}$. Hence, $y_{2 i+1}$ and $y_{2 j+1}$ have the same level, there is a $k$ such that $h^{k}\left(y_{2 i+1}\right)=h^{k}\left(y_{2 j+1}\right)=z$.

Assume $i<j$. It will be shown that $y_{2 i+2} \in C_{2}$. This will give the desired contradiction and prove the claim. Note that $y_{2 i+1}<_{l} y_{2 i+2}<_{l} y_{2 j+1}$. Hence $h^{k}\left(y_{2 i+1}\right)$ $\leqq_{l} h^{k}\left(y_{2 i+2}\right) \varliminf^{k}\left(y_{2 j+1}\right)$, so $z \leqq_{l} h^{k}\left(y_{2 i+\cdot 2}\right) \varliminf_{l} z$. It follows that $h^{k}\left(y_{2 i+2}\right)=z$.

Now consider the set $D_{x}=\left\{y_{1}, y_{3}, \ldots, y_{2 k+1}\right\}$. Since $y_{2 i+2} \notin D_{x}$ and $D_{x} \cup\left\{y_{2 i+2}\right\}$ is level, $y_{2 i+2} \mid D_{x}$. Also $z \in \hat{h}\left(y_{2 i+2}\right) \cap D_{g(x)}$, so $\hat{h}\left(y_{2 i+2}\right) \cap D_{g(x)} \neq \varnothing$. Thus $x$ witnesses that $y_{2 i+2} \in C_{2}$, which was to be shown.

Let $b_{0}, b_{1}, \ldots$ be the members of $B$ in increasing order. Since $B$ is not hyperimmune, it follows from [14, Theorem 21] that there is a recursive function $f$ such that $f(k)>b_{k}$ for all $k$. We now define a recursive sequence of sets $D_{p(k)}$ all intersecting $B$. To find $D_{p(k)}$, list $C$ until there are at most $2 n$ numbers of level $k$ which are less than $f(k)$ and have not yet appeared in $C$. By the previous argument, this state of affairs must eventually be reached for every $k$. Then let $D_{p(k)}$ be those numbers $<f(k)$ of level $k$ which have not yet appeared in $C$ when this state of affairs is reached. Thus $\left|D_{p(k)}\right| \leqq 2 n$ for all $k$. Also $D_{p(k)} \cap B \neq \varnothing$ because $b_{k} \in D_{p(k)}$ $\cap B$. Finally the $D_{p(k)}$ are pairwise disjoint because the levels are. However, 
Theorem 2 of [1] states that no immune retraceable set is witnessed nonhyperimmune by an array (such as $D_{p(k)}$ ) of sets of bounded cardinality. This contradiction completes the proof.

We remark as in $\$ 2$ that the conclusion of Theorem 6 fails even for $n=1$ when $B$ is permitted to be hyperimmune and indeed whenever $B$ is the complement of some hypersimple set $A$ obtained by Dekker's hypersimple set construction. On the other hand, if $\mathscr{A}^{2} \leqq_{m} \mathscr{A}^{1}$ where $B=\bar{A}$ is retraceable, then $A$ must be r.e. [5, Corollary 4.29]. It now follows from [3, Theorem 4] that $\mathscr{A}^{1}<_{m} \mathscr{A}^{2}$ when $\bar{A}$ is immune, nonhyperimmune, and retraceable. We conjecture that under these assumptions $\mathscr{A}^{n}<_{m} \mathscr{A}^{n+1}$ for all $n$, so that the existence of a general recursive retracing function is not necessary in Theorem 6.

COROLlary 7. Each nonrecursive tt-degree contains an infinite chain of $m$-degrees.

Proof. We need to know that each nonrecursive $t t$-degree contains an immune nonhyperimmune set $B$ retraced by a general recursive function. This result is implicit in [3]. Suppose we are given a set $E$. Let $\left\{a_{n}\right\}$ be the sequence of values of the characteristic function of $E$ and let $\sigma=\Phi\left\langle a_{n}\right\rangle$ be the set constructed from $\left\{a_{n}\right\}$ on p. 363 of [3]. Then it is pointed out in [3] that if $E$ is nonrecursive, then $\sigma$ is immune but not hyperimmune. Further, it is easy to check that $\sigma \equiv_{t t} E$ and $\sigma$ is retraced by a general recursive function. Hence, if we let $B=\sigma$ in the theorem, we see that $\mathscr{A}^{n}<_{m} \mathscr{A}^{n+1}$, where $A=\bar{B}$. Also we have $E \equiv_{t t} \sigma \equiv_{t t} A \equiv_{t t} \mathscr{A}^{n}$ for all $n$.

The author does not know whether Corollary 7 holds for infinite antichains, but the existence of a pair of incomparable $m$-degrees within each $t t$-degree is proved in [6, Corollary 4.3(iv)].

4. $t t$-degrees within $T$-degrees. Martin has communicated to the author a sufficient condition for a $T$-degree to consist of a single $t$-degree. In the following theorem we show that his condition is also necessary and deduce that each $T$-degree either consists of a single $t t$-degree or contains an infinite chain of $t t$-degrees.

THEOREM 8. If $a$ is any T-degree, the following statements are equivalent:

(i) Every function $f$ of degree $\leqq a$ is (everywhere) majorized by some recursive function.

(ii) a consists of a single tt-degree.

(iii) a has a finite number of tt-degrees.

(iv) There is a maximum tt-degree among the tt-degrees contained in a.

Proof. (We remark that by Corollary 1.1 of [10], (i) is equivalent to the assertion that no hyperimmune set has degree $a$. However, we shall not need this fact.)

In this proof we write $\{e\}^{C}$ for the $e$ th function partial recursive in the set $C$. We write $\{e\}_{s}^{C}(x)=y$ if at most $s$ machine steps are necessary to compute $\{e\}^{C}(x)=y$. $\{e\}$ and $\{e\}_{s}$ are $\{e\}^{\varnothing},\{e\}_{s}^{\varnothing}$ respectively. We also identify sets and their characteristic functions. 
The implication (i) $\Rightarrow$ (ii) is due to Martin, but we give it here because it is unpublished. Assume (i). Let $A$ be a set of degree $a$ and assume $B \leqq_{T} A$. We wish to show $B \leqq_{t t} A$. Assume $B=\{e\}^{A}$. Define $f(x)=\mu s$ [ $\{e\}_{s}^{A}(x)$ is defined]. Then $f$ is recursive in $A$, so by (i) there is a recursive function $g$ such that for all $x, g(x)>f(x)$. Define a new recursive reduction procedure as follows:

$$
\begin{aligned}
\left\{e^{\prime}\right\}^{C}(x) & =\{e\}_{g(x)}^{C}(x) & & \text { if }\{e\}_{(g x)}^{C}(x) \text { defined, } \\
& =0 & & \text { otherwise }
\end{aligned}
$$

Then $B=\left\{e^{\prime}\right\}^{A}$ also and $\left\{e^{\prime}\right\}^{C}$ is a total function for every set $C$. It follows by a theorem of Nerode [12, p. 250] or [15, p. 143] that $B \leqq_{t t} A$. Thus if $B \equiv_{T} A$, we may also apply this argument with $A$ and $B$ interchanged and deduce that $B \equiv_{t t} A$. Hence (ii) holds.

The implications (ii) $\Rightarrow$ (iii) and (iii) $\Rightarrow$ (iv) are trivial.

We now show that (iv) $\Rightarrow$ (i). Assume that (iv) is true but (i) is false. Thus $a$ has a maximum $t t$-degree, and within this $t t$-degree there is a maximum $m$-degree by [15, p. 113]. Let $A$ be a set belonging to the maximum $m$-degree in $a$. Also, let $f$ be a function recursive in $a$ which is majorized by no recursive function. We may assume that $f$ is monotone increasing. We use a diagonal construction similar to that in Theorem I of Chapter 9 of [15]. Let

$$
B=\left\{e:\{e\}_{f(e)}(e) \text { defined } \&\{e\}_{f(e)}(e) \notin A\right\} .
$$

Clearly, $B \leqq_{T} A$. We claim $B \varliminf_{m} A$. Assume $B \leqq_{m} A$ via $\left\{e_{0}\right\}$. Assume that $e_{0}, e_{1}, \ldots$ is a recursive sequence such that $\left\{e_{i}\right\}=\left\{e_{0}\right\}$ and $e_{i}>i$ for all $i$. Thus we have for all $i, e_{i} \in B \Leftrightarrow\left\{e_{i}\right\}\left(e_{i}\right) \in A$. Then we see from the definition of $B$ that $\left\{e_{i}\right\}_{\left(f\left(e_{i}\right)\right)}\left(e_{i}\right)$ is defined for no $i$. Thus if we define $g(i)=\mu s\left[\left\{e_{i}\right\}_{s}\left(e_{i}\right)\right.$ is defined], we have $g(i) \geqq f\left(e_{i}\right)$ $>f(i)$. But since $g$ is recursive, this contradicts the selection of $f$. Hence $B \$_{m} A$.

Let $C=\{2 n: n \in A\} \cup\{2 n+1: n \in B\}$. Clearly $C \equiv_{T} A$ and $A<_{m} C$. This contradicts the selection of $A$.

COROLlaRY 9. (i) If a T-degree consists of a single tt-degree, then so do all lower T-degrees.

(ii) Each T-degree either consists of a single tt-degree or contains an infinite chain of tt-degrees.

Proof. Part (i) of the corollary holds because if (i) of the theorem is true of $a$, it is also true of all $T$-degrees $\boldsymbol{b} \leqq a$. (ii) of the corollary follows immediately from (ii) $\Leftrightarrow$ (iv) in the theorem.

We remark that Miller and Martin [10, Theorem 2.1] have shown the existence of nonrecursive $T$-degrees which have no hyperimmune sets and hence consist of a single $t t$-degree. On the other hand, Martin has shown $[9, \S 2$, Corollary 1] that the $T$-degrees of hyperimmune sets have measure 1 so that "almost every" $T$ degree contains an infinite chain of $t t$-degrees. 


\section{REFERENCES}

1. K. I. Appel and T. G. McLaughlin, On properties of regressive sets, Trans. Amer. Math. Soc. 115 (1965), 83-93.

2. J. C. E. Dekker, A theorem on hypersimple sets, Proc. Amer. Math. Soc. 5 (1954), 791-796.

3. J. C. E. Dekker and J. Myhill, Retraceable sets, Canad. J. Math. 10 (1958), 357-373.

4. P. C. Fischer, A note on bounded-truth-table reducibility, Proc. Amer. Math. Soc. 14 (1963), 875-877.

5. C. G. Jockusch, Jr., Reducibilities in recursive function theory, Doctoral Thesis, Massachusetts Institute of Technology, Cambridge, 1966.

6. - Semirecursive sets and positive reducibility; Trans. Amer. Math. Soc. 131 (1968), $420-436$.

7. A. H. Lachlan, Many-one degrees (to appear).

8. M. Lerman, Recursive functions modulo co-maximal sets, Doctoral Thesis, Cornell Univ., Ithaca, N. Y., 1968.

9. D. A. Martin, Measure, category, and degrees of unsolvability (unpublished).

10. W. Miller and D. A. Martin, The degrees of hyperimmune sets, Z. Math. Logik Grundlagen Math. 14 (1968), 159-166.

11. J. Myhill, Creative sets, Z. Math. Logik Grundlagen Math. 1 (1955), 97-108.

12. A. Nerode, General topology and partial recursive functionals, Summaries of talks presented at the Summer Institute for Symbolic Logic, Cornell University, 1957, pp. 247-251.

13. E. L. Post, Recursively enumerable sets of integers and their decision problems, Bull. Amer. Math. Soc. 50 (1944), 284-316.

14. H. G. Rice, Recursive and recursively enumerable orders, Trans. Amer. Math. Soc. 83 (1956), 277-300.

15. H. Rogers, Jr., Theory of recursive functions and effective computability, McGraw-Hill, New York, 1967.

16. R. I. Soare, Recursion theory and Dedekind cuts, Trans. Amer. Math. Soc. 140 (1969), 271-294.

17. C. E. M. Yates, Three theorems on the degrees of recursively enumerable sets, Duke Math. J. 32 (1965), 461-468.

18. P. R. Young, Linear orderings under one-one reducibility, J. Symbolic Logic 31 (1966), $70-85$.

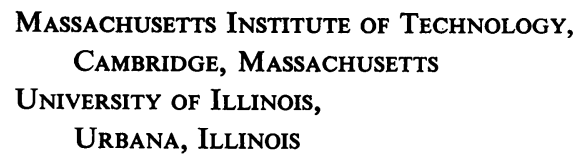

